\title{
Ações do enfermeiro na identificação precoce de alterações sistêmicas causadas pela sepse grave
}

\section{Actions of nurses in early identification of systemic changes caused by severe sepsis}

Felipe Garrido ${ }^{1}$, Luana Tieppo ${ }^{1}$, Maria Dalva da Silva Pereira ${ }^{1}$, Rodrigo de Freitas ${ }^{1}$, Welington Maciel de Freitas ${ }^{1}$, Rosangela Filipini², Patricia Granja Coelho ${ }^{3}$, Fernando Luiz Affonso Fonseca ${ }^{4,5}$, Ana Maria Marcondes Fiorano ${ }^{2}$

${ }^{1}$ Curso de Especialização em Enfermagem, Cuidados Críticos, Faculdade de Medicina do ABC (FMABC) - Santo André (SP), Brasil.

${ }^{2}$ Curso de Enfermagem, FMABC - Santo André (SP), Brasil.

${ }^{3}$ Cursos de Saúde e Bem-Estar, Serviço Nacional de Aprendizagem Comercial (SENAC) - São Paulo (SP), Brasil.

${ }^{4}$ Curso de Gestão em Saúde Ambiental, FMABC - Santo André (SP), Brasil.

Instituto de Ciências Ambientais, Químicas e Farmacêuticas, Universidade Federal de São Paulo (UNESP) - Diadema (SP), Brasil.

DOI: http://dx.doi.org/10.7322/abcshs.v42i1.944

\begin{abstract}
RESUMO
Introdução: As unidades de terapia intensiva (UTIs) têm sido organizadas como setores estratégicos para o suporte especializado de assistência ao paciente grave. Objetivo: Verificar as ações do enfermeiro para a identificação precoce das alterações sistêmicas causadas pela sepse grave relacionadas às alterações hemodinâmicas, neurológicas, respiratórias, renais e nutricionais dos pacientes internados em UTIs adulto. Métodos: Estudo descritivo com 24 enfermeiros. Os dados foram coletados por meio de formulário composto de questões estruturadas. Resultados: Apenas 36\% dos enfermeiros possuem especialização em UTI adulto; verificou-se que os profissionais identificam parcialmente os sinais e sintomas apresentados pelo paciente séptico. Conclusão: Os enfermeiros encontram dificuldade na identificação precoce das alterações sistêmicas causadas pela sepse grave relacionada às alterações hemodinâmicas, neurológicas, respiratórias, renais e nutricionais dos pacientes internados em UTI adulto, o que pode estar relacionado com a falta de treinamento e de protocolos estabelecidos pelas instituições.
\end{abstract}

Palavras-chave: unidade de terapia intensiva; sepse; síndrome de resposta inflamatória sistêmica; enfermagem.

\begin{abstract}
Introduction: Intensive care units (ICUs) have been organized as strategic sectors for specialized support assistance to critically ill patients. Objective: To assess the actions of the nurse for the early identification of systemic changes caused by severe sepsis, related to hemodynamic, neurological, respiratory, renal and nutrition changes of patients in adult ICUs. Methods: Descriptive studies with 24 nurses. Data were collected using a questionnaire composed of structured questions. Results: Only $36 \%$ of nurses have expertise in adult ICUs, professionals partially identify the signs and symptoms presented by the septic patient. Conclusion: Nurses find difficulty in the early identification of systemic changes caused by severe sepsis, related to hemodynamic, neurological, respiratory, renal and nutritional changes seen in patients in adult ICUs, which may be related to lack of training and of protocols established by the institutions.
\end{abstract}

Keywords: intensive care unit; sepsis; systemic inflammatory response syndrome; nursing. 


\section{INTRODUÇÃO}

As unidades de terapia intensiva (UTIs) têm sido organizadas como setores estratégicos para o suporte especializado de assistência ao paciente grave, envolvendo o uso de recursos tecnológicos e terapêuticos de ponta.

Contudo, entre todas as doenças que acometem pacientes críticos, a sepse, o choque séptico e a disfunção de múltiplos órgãos são as maiores causas de morte nas UTIs ${ }^{1,2}$.

Apesar da realização da Conferência de Consenso, existem muitas controvérsias quanto ao modo ideal de diagnóstico precoce da sepse, persistindo a dificuldade na categorização e na padronização diagnósticas ${ }^{2}$.

A sepse é caracterizada pela ocorrência de uma reação inflamatória sistêmica, a síndrome da resposta inflamatória sistêmica (SIRS), com um foco infeccioso presumido ou evidente. A associação de sepse com disfunção orgânica caracteriza a sepse grave ${ }^{3}$.

A sepse grave e o choque séptico associam-se a uma alta mortalidade e a custos hospitalares elevados. No Brasil, estudos recentes sobre a sepse grave apontam que a doença é responsável por $54,5 \%$ da taxa de letalidade ${ }^{3,4}$.

A publicação das diretrizes que serviram de base para o lançamento da campanha internacional Surviving Sepsis Campaign, em 2004, definiu um programa internacional desenhado para conscientizar, planejar e disseminar as ações de práticas clínicas no tratamento da sepse.

Desde 2005, após uma revisão desse programa, apareceram os chamados Severe Sepsis Bundles, uma série de atividades e tratamentos esquematizados para que as equipes que atendem esses pacientes sigam os tempos, as sequências e os objetivos dos componentes individuais, com a meta final de reduzir a mortalidade por sepse grave em $25 \%{ }^{2}$.

Entre os comprometimentos clínicos decorrentes da sepse grave que devem ser observados e avaliados pelo enfermeiro, a lesão renal aguda (LRA) aparece em $23 \%$ dos pacientes e em $51 \%$ dos pacientes com choque séptico ${ }^{5}$.

A LRA ocorre com a redução súbita da filtração glomerular e o aumento dos níveis de creatinina sérica. O mecanismo de isquemia renal é descrito como a principal causa de LRA associada à sepse, dada a hipoperfusão que induz à lesão das células do epitélio tubular, apoptose e necrose tubular aguda ${ }^{5}$.

Com a falência renal, ocorrem distúrbios funcionais importantes que também afetam a homeostase metabólica. Assim, nesse quadro podem ocorrer alterações como letargia, confusão mental, desorientação, delirium, torpor e coma, os quais podem estar presentes ou se manifestar em decorrência de hipoxemia ou acidose ${ }^{6}$.

Essas manifestações clínicas também se tornam evidentes na vigência de distúrbios neurológicos secundários à sepse, haja vista a diminuição da perfusão cerebral como disfunção orgânica associada.

Igualmente relevante, a disfunção respiratória na sepse deflagra um quadro clínico grave, que determina a inadequada troca gasosa pela diminuição da complacência pulmonar, provocada por excesso de líquidos no espaço intersticial ${ }^{7}$.

Outro aspecto importante entre os pacientes sépticos está relacionado à subnutrição, deficiência que resulta em alterações imunológicas. Essas, por sua vez, aumentam os riscos de infecções, a permanência hospitalar e as taxas de mortalidade, elevando, consequentemente, os custos na área da saúde ${ }^{8,9}$.

A UTI inclui pacientes em resposta de fase aguda e requer uma abordagem múltipla que visa controlar o processo infeccioso e promover suporte metabólico ${ }^{8,9}$.

Contudo, existe grande heterogeneidade no grau de seguimento dessas recomendações. Nesse contexto, as habilidades e competências do profissional de enfermagem acompanham a importante responsabilidade de avaliar criteriosamente o paciente e participar do processo de tomada de decisões com a equipe multiprofissional, no sentido de garantir o trabalho em equipe, de acordo com as recomendações do protocolo atual diante dos quadros de sepse.

Diante do exposto, este estudo teve o objetivo de verificar as ações do enfermeiro para identificação precoce das alterações sistêmicas causadas pela sepse grave relacionada às alterações hemodinâmicas, neurológicas, respiratórias, renais e nutricionais dos pacientes internados em UTIs adulto.

\section{MÉTODO}

Trata-se de estudo quantitativo, descritivo simples, desenvolvido na UTI de quatro hospitais públicos com administração indireta na região do ABCD Paulista.

A coleta de dados ocorreu durante o mês de outubro de 2013, por meio de visitas ao setor de UTI durante os plantões diurno e noturno. A amostra foi composta de 24 enfermeiros de ambos os sexos. Enfermeiros que atuavam em UTI adulto durante os plantões diurno e noturno configuraram o critério de inclusão.

Como o número de participantes não foi estabelecido previamente, a coleta dos dados ocorreu com todos os profissionais que aceitaram participar do estudo.

Foi aplicado um pré-teste para análise da clareza do instrumento, antes da efetivação da coleta de dados. Esse teste sofreu adequações na medida em que foram percebidas necessidades de reformulação das perguntas. Constatada a versão que traduziu a melhor interpretação das questões, o instrumento foi dado como finalizado e aplicado a todos os sujeitos do estudo.

Os dados foram coletados nas próprias instituições, em local apropriado e disponibilizado pelo responsável técnico pelos serviços. No momento da entrevista foi aplicado o instrumento com questões de caracterização dos profissionais e questões estruturadas, que abordaram o manejo da sepse grave em atenção aos sistemas neurológico, respiratório, hemodinâmico, renal e nutricional.

O processamento e a análise dos dados foram feitos por meio do software Epi-Info 6.0 e análise univariada, com nível de significância em $5 \%$. 
Quanto aos aspectos éticos, a coleta de dados foi iniciada somente após aprovação do Comitê de Ética em Pesquisa, em 25 de setembro de 2013, sob o no 420.187.

Previamente, os sujeitos do estudo foram esclarecidos quanto ao objetivo da pesquisa e à sua participação voluntária, e tiveram a garantia de sigilo de suas respostas por meio do Termo de Consentimento Livre e Esclarecido.

\section{RESULTADOS}

Foram entrevistados 24 enfermeiros atuantes em UTI na região do ABC no mês de outubro de 2013. Observou-se um predomínio do sexo feminino (54,16\%); quanto à idade, a média observada foi, respectivamente, de 33,8 e 33,5 anos para homens e mulheres. No entanto, verificou-se um desvio padrão de 6,7 e 7,8 anos, respectivamente, evidenciando que há uma grande variabilidade nas idades.

Os profissionais entre 35 e 49 anos apresentam maior tempo médio de atuação profissional como enfermeiro (Tabela 1); situação também observada quanto à experiência profissional em UTI, sendo esses dados estatisticamente significantes $(\mathrm{p}=0,009 ; \mathrm{p}=0,005)$.

Na Tabela 2 observa-se que apenas 9 (36\%) enfermeiros possuem especialização em UTI e 3 (12\%) referem ainda cursar a pós-graduação. Por outro lado, 12 (48\%) não referiram especialização nessa área, mas em outras. Apenas 2 (8\%) não referiram especialização alguma.

Também na Tabela 2 observa-se que, quando questionados sobre a utilização de protocolos para o manejo da sepse, 13 (52\%) profissionais afirmaram que em suas instituições de trabalho não há essa aplicação e 12 (48\%) afirmaram não ter dificuldades nessa atividade.

Na Tabela 3 observa-se que, na avaliação inicial do paciente séptico, apenas 2 ( $8 \%$ ) enfermeiros consideram o percentual de bastonetes na análise do hemograma, bem como a contagem de leucócitos, haja vista que 3 (12\%) relataram a observação de leucopenia.

Tabela 1: Relação entre sexo, idade e tempo de atuação do enfermeiro em unidade de terapia intensiva. Santo André, 2013

\begin{tabular}{|c|c|c|c|c|c|c|}
\hline Variáveis & $\mathbf{n}$ & $\begin{array}{l}\text { Média } \\
\text { idade }\end{array}$ & $\begin{array}{l}\text { Mín./ } \\
\text { Máx. }\end{array}$ & DP & K2 & Valor $p$ \\
\hline \multicolumn{7}{|l|}{ Sexo } \\
\hline Masc. & 11 & 33,8 & $24 / 44$ & 6,7 & 0,021 & $0,885^{*}$ \\
\hline Fem. & 13 & 33,5 & $22 / 49$ & 7,8 & & \\
\hline $\begin{array}{l}\text { Idade } \\
\text { (anos) }\end{array}$ & & $\begin{array}{l}\text { Tempo de } \\
\text { atuação }\end{array}$ & & & & \\
\hline 22-34 & 12 & 3,4 & $6,2 / 10$ & 3,3 & 8,1 & $0,009^{* *}$ \\
\hline $35-49$ & 12 & 7,6 & 03/16 & 3,9 & & \\
\hline $\begin{array}{l}\text { Idade } \\
\text { (anos) }\end{array}$ & & $\begin{array}{c}\text { Tempo de } \\
\text { atuação em UTI }\end{array}$ & & & & \\
\hline 22-34 & 12 & 1,2 & $0,1 / 4$ & 1,6 & 7,7 & $0,005^{\star}$ \\
\hline 35-49 & 12 & 4,2 & $0,5 / 10$ & 3,3 & & \\
\hline
\end{tabular}

DP: desvio padrão; *teste de análise de variância; **teste de Kruskal-Wallis; UTI: unidade de terapia intensiva.
Ainda quanto ao exame inicial, 24 (96\%) profissionais atentaram para temperatura, 17 (68\%) para frequência cardíaca e 15 (60\%) para leucocitose. Apenas 13 (52\%) identificam a frequência respiratória maior do que 20 movimentos por minuto na avaliação do paciente em sepse.

Em relação à avaliação neurológica, 23 (92\%) enfermeiros procuram identificar o rebaixamento do nível de consciência como alteração do sistema nervoso central, seguido de alteração do padrão respiratório (18 a 72\%) e confusão mental (17 a 68\%).

$\mathrm{Na}$ avaliação respiratória verificou-se que 21 (84\%) enfermeiros atentaram para alteração do padrão respiratório, 15 (60\%) para aspecto e quantidade da secreção traqueal e também para frequência respiratória maior do que 20 respirações por minuto, $14(56 \%)$ para saturação de oxigênio menor do que $80 \%$, bem como para presença de leucocitose/leucopenia. Apenas 3 (12\%) observam a relação da pressão parcial do oxigênio no sangue arterial $\left(\mathrm{PaO}_{2}\right)$ e elevação da fração inspirada de oxigênio $\left(\mathrm{FIO}_{2}\right)$ menor do que 250.

Em relação à avaliação renal, 21 (84\%) percebem a oligúria como indicação da perda de função dos rins. Quanto à avaliação da função renal, 11 (44\%) profissionais observaram os valores de ureia e 12 (48\%) verificaram os níveis de creatinina; 6 (24\%) enfermeiros consideram o balanço hídrico positivo como indicador de falência renal. Ainda, apenas 10 (40\%) observam sinais clínicos, como letargia, torpor e coma, como indicadores de alteração da função renal.

Tabela 2: Distribuição dos profissionais quanto à formação, atuação em unidade de terapia intensiva e utilização do protocolo para sepse. Santo André, 2013

\begin{tabular}{|l|c|}
\hline Variáveis & $\mathbf{n}(\%)$ \\
\hline Especialização & \\
\hline Sim & $9(36,0)$ \\
\hline Não & $12(48,0)$ \\
\hline Cursando & $3(12,0)$ \\
\hline Área de atuação & \\
\hline Centro cirúrgico & $2(8,0)$ \\
\hline Cardiologia & $4(16,0)$ \\
\hline Comissão de Controle de Infecção Hospitalar & $1(4,0)$ \\
\hline Emergência & $2(8,0)$ \\
\hline Oncologia & $1(4,0)$ \\
\hline Outros & $2(8,0)$ \\
\hline Não procede & $2(8,0)$ \\
\hline Uso de protocolo para sepse na instituição & \\
\hline Sim & $10(40,0)$ \\
\hline Não & $13(52,0)$ \\
\hline Dificuldades em aplicar protocolo & \\
\hline Sim & $7(28,0)$ \\
\hline Não & $12(48,0)$ \\
\hline Dificuldades com protocolo & \\
\hline Diferentes condutas médicas & $1(4,0)$ \\
\hline Avaliação nutricional & $1(4,0)$ \\
\hline Espaço físico dificulta a proximidade & $1(4,0)$ \\
\hline Não está implantado & $1(4,0)$ \\
\hline Não há impresso específico & $1(4,0)$ \\
\hline
\end{tabular}

Obs.: Os dados ignorados foram excluídos. 
Quanto à avaliação nutricional, apenas 9 (36\%) enfermeiros observaram que os níveis glicêmicos devem ser menores do que $150 \mathrm{mg} / \mathrm{dL}$ e 14 (56\%) identificaram a necessidade de início precoce do suporte nutricional. A maioria dos sujeitos parece não conhecer o aporte calórico dos pacientes sépticos, pois de 12 a $28 \%$ consideraram o protocolo de suporte nutricional.

Tabela 3: Distribuição das ações de enfermeiros atuantes em unidade de terapia intensiva de acordo com o protocolo de sepse. Santo André, 2013

\begin{tabular}{|c|c|}
\hline Variável & n (\%) \\
\hline \multicolumn{2}{|l|}{ Infecção na avaliação inicial, o enfermeiro identifica } \\
\hline $\mathrm{T}>38^{\circ} \mathrm{C}$ ou $<36^{\circ} \mathrm{C}$ & $24(96,0)$ \\
\hline $\mathrm{FC}>90 \mathrm{bpm}$ & $17(68,0)$ \\
\hline $\mathrm{FR}>20 \mathrm{mpm}$ & $13(52,0)$ \\
\hline $\mathrm{PACO}_{2}<32 \mathrm{mmHg}$ & $5(20,0)$ \\
\hline Leucocitose $\left(>12.000 \mathrm{~mm}^{3}\right)$ & $15(60,0)$ \\
\hline Leucopenia $\left(<4.000 \mathrm{~mm}^{3}\right)$ & $3(12,0)$ \\
\hline $10 \%$ bastonetes & $2(8,00)$ \\
\hline Rebaixamento nível de consciência & $23(92,0)$ \\
\hline Convulsões & $2(8,0)$ \\
\hline Confusão mental & $17(68,0)$ \\
\hline Delirium & $5(20,0)$ \\
\hline Agitação psicomotora & $7(28,0)$ \\
\hline Alteração padrão respiratório & $18(72,0)$ \\
\hline \multicolumn{2}{|l|}{$\mathrm{Na}$ avaliação nutricional, observa } \\
\hline Início precoce aporte nutrientes em até $72 \mathrm{~h}$ & $14(56,0)$ \\
\hline Glicemia abaixo $250 \mathrm{mg} / \mathrm{dL}$ & $9(36,0)$ \\
\hline \multicolumn{2}{|l|}{ Aporte calórico/proteico } \\
\hline $25-27 \mathrm{kcal} / \mathrm{kg}$ e $1,5 \mathrm{~g}$ prot $/ \mathrm{kg}$ & $7(28,0)$ \\
\hline \multicolumn{2}{|l|}{ Fase aguda } \\
\hline $20-25 \mathrm{kcal} / \mathrm{kg}$ e $1,5-2 \mathrm{~g} \mathrm{prot} / \mathrm{kg}$ & $3(12,0)$ \\
\hline \multicolumn{2}{|l|}{ Desnutrição } \\
\hline $25-30 \mathrm{kcal} / \mathrm{kg} \mathrm{e} 1,5-2 \mathrm{~g} \mathrm{prot} / \mathrm{kg}$ & $4(16,0)$ \\
\hline \multicolumn{2}{|l|}{ Obesos críticos $\left(\mathrm{IMC}>30 \mathrm{~kg} / \mathrm{m}^{2}\right)$} \\
\hline $11-14 \mathrm{kcal}$ peso atual ou & \multirow{2}{*}{$3(12,0)$} \\
\hline $22-25 \mathrm{kcal}$ peso ideal & \\
\hline \multicolumn{2}{|l|}{$\mathrm{Na}$ avaliação respiratória, observa } \\
\hline Alteração padrão respiratório & $21(84,0)$ \\
\hline Secreção traqueal & $15(60,0)$ \\
\hline Ruídos adventícios & $11(44,0)$ \\
\hline Alterações de expansibilidade & $6(24,0)$ \\
\hline Frequência respiratória>20 & $15(60,0)$ \\
\hline Sat $\mathrm{O}_{2}<80 \%$ & $14(56,0)$ \\
\hline $\mathrm{PCO}_{2}<32 \%$ & $7(28,0)$ \\
\hline $\mathrm{PAO}_{2} \mathrm{FIO}_{2}<250$ & $3(12,0)$ \\
\hline Leucocitose/leucopenia & $14(56,0)$ \\
\hline \multicolumn{2}{|l|}{$\mathrm{Na}$ avaliação renal, observa } \\
\hline Oligúria & $21(84,0)$ \\
\hline Hipoperfusão renal & $13(52,0)$ \\
\hline Creatinina $>1,2 \mathrm{mg} / \mathrm{dL}$ & $12(48,0)$ \\
\hline Ureia $>45 \mathrm{mg} / \mathrm{dL}$ & $11(44,0)$ \\
\hline Letargia, torpor, convulsão, coma & $10(40,0)$ \\
\hline Náuseas, vômito, diarreia & $4(16,0)$ \\
\hline Poliúria & $1(4,0)$ \\
\hline Balanço hídrico positivo & $6(24,0)$ \\
\hline
\end{tabular}

IMC: índice de massa corporal. FC: frequência cardíaca. FR: frequência respiratória. $\mathrm{PACO}_{2}$ : pressão de $\mathrm{CO}_{2}$
Quanto às providências em acordo com o protocolo de sepse (Tabela 4), observa-se que 14 (56\%) afirmaram que estas ocorrem nos primeiros 60 minutos após o diagnóstico médico, 5 (20\%) reportaram que a pressão venosa central (PVC) deve se manter em torno de $8 \mathrm{mmHg}, 6$ (24\%) referiram que a hemoglobina deve permanecer maior do que $10 \mathrm{~g} / \mathrm{dL}, 5(20 \%)$ relataram que a saturação venosa de $\mathrm{O}_{2}\left(\mathrm{SpVO}_{2}\right)$ deve permanecer em torno de $70 \%$ e 3 (12\%) citaram que o lactato deve ser menor do que $2 \mathrm{mmol}$.

\section{DISCUSSÃO}

Este estudo evidenciou que os enfermeiros parecem apresentar dificuldades em utilizar protocolos para assistência a pacientes em sepse, provavelmente devido a razões institucionais, como a falta de impressos específicos ou até mesmo a ausência dessa prática no setor. Outro motivo que pode implicar na subutilização das recomendações para o atendimento do paciente séptico consiste na dificuldade de interpretação dos dados clínicos do paciente pelo enfermeiro, podendo estar relacionado ou não com a falta de treinamento e o envolvimento das instituições nas ações do enfermeiro na sepse.

As lesões endoteliais disseminadas no paciente com sepse grave resultam em grandes perdas hídricas para o espaço intersticial, o

Tabela 4: Providências tomadas pelo enfermeiro de acordo com o protocolo de sepse. Santo André, 2013

\begin{tabular}{|c|c|}
\hline Providência & n (\%) \\
\hline \multicolumn{2}{|l|}{ Tempo } \\
\hline $30 \mathrm{~min}$ & $7(28,0)$ \\
\hline $60 \mathrm{~min}$ & $14(56,0)$ \\
\hline $120 \mathrm{~min}$ & $3(12,0)$ \\
\hline \multicolumn{2}{|l|}{ Manter PVC } \\
\hline$>5 \mathrm{mmHg}$ & $4(16,0)$ \\
\hline$>7 \mathrm{mmHg}$ & $3(12,0)$ \\
\hline$>8 \mathrm{mmHg}$ & $5(20,0)$ \\
\hline$>12 \mathrm{mmHg}$ & $10(40,0)$ \\
\hline \multicolumn{2}{|l|}{ Manter $\mathrm{Hb}$} \\
\hline$>9$ & $6(24,0)$ \\
\hline$>10$ & $6(24,0)$ \\
\hline$>11$ & $6(24,0)$ \\
\hline \multicolumn{2}{|c|}{ Manter PAM nas primeiras 6 horas } \\
\hline$>50 \mathrm{mmHg}$ & $8(32,0)$ \\
\hline$>60 \mathrm{mmHg}$ & $10(40,0)$ \\
\hline$>70 \mathrm{mmHg}$ & $2(8,0)$ \\
\hline$>80 \mathrm{mmHg}$ & $4(16,0)$ \\
\hline \multicolumn{2}{|c|}{ Manter $\mathrm{SPVO}_{2}$ nas primeiras 6 horas } \\
\hline $50 \%$ & $6(24,0)$ \\
\hline $70 \%$ & $5(20,0)$ \\
\hline $65 \%$ & $1(4,0)$ \\
\hline \multicolumn{2}{|l|}{ Manter o lactato } \\
\hline$<2 \mathrm{mmol}$ & $3(12,0)$ \\
\hline$>4 \mathrm{mmol}$ & $2(8,0)$ \\
\hline$>10 \%$ valor inicial coletado & $11(44,0)$ \\
\hline
\end{tabular}

PVC: pressão venosa central; PAM: pressão arterial média; $\mathrm{SVPO}_{2}$ : saturação de oxigênio. 
que causa o comprometimento do fluxo sanguíneo e, consequentemente, isquemia de extensos territórios, levando à disfunção de múltiplos órgãos e elevando a chance de óbito ${ }^{10}$.

A terapia precoce guiada por metas busca atingir a readequação da oferta de oxigênio aos tecidos, evitando, assim, a disfunção de múltiplos órgãos. É indicada quando o paciente com sepse grave apresenta o lactato alterado e hipotensão refratária. Manter os valores preconizados para a PVC, a pressão arterial média (PAM), o débito urinário e a saturação venosa central de oxigênio é uma meta que deve ser alcançada em um prazo de seis horas ${ }^{11}$.

$\mathrm{O}$ aumento do lactato é muito rápido, proporcional ao defeito oxidativo do metabolismo e à gravidade do choque; assim, a dosagem do lactato sérico é de grande importância. Pacientes com hiperlactatemia apresentam quase 2,5 vezes mais chances de evoluir para óbito. Entretanto, o dado isolado do lactato não auxilia na avaliação da resposta terapêutica nem na probabilidade de sobrevida ${ }^{11}$.

É de suma importância para a redução da mortalidade na sepse grave que seja realizado o conjunto de ações descritas. Quanto maior a eficácia das ações, com a qualidade requerida, maior o sucesso na reversão e/ou promoção da melhora do quadro. Portanto, para que essas ações sejam tomadas é necessário que o enfermeiro tenha conhecimento técnico-científico para identificar corretamente as alterações nos valores de $\mathrm{PAM}, \mathrm{PVC}, \mathrm{SvCO}_{2}$ e lactato sérico. Além disso, deve-se reconhecer o tempo para tomada de decisões, o qual é essencialmente importante, uma vez que incide diretamente no quadro do paciente ${ }^{11}$.

Observou-se, neste estudo, que os enfermeiros estão mais atentos à avaliação da PAM e da PVC. Assim sendo, é necessário que as dificuldades para a análise clínica dos demais indicadores, como valores de $\mathrm{SvCO}_{2}$ e lactato, sejam superadas, a fim de promoverem uma assistência mais bem qualificada, apoiada integralmente nas recomendações dos protocolos preconizados, garantindo, consequentemente, mais eficiência e eficácia no manejo dos pacientes sépticos.

No que se refere à avaliação neurológica, a disfunção cerebral na sepse é precoce, sendo muitas vezes a primeira disfunção orgânica a se manifestar; o cérebro é o órgão que possui maior consumo de $\mathrm{O}_{2}$ por g de tecido e possui poucas defesas antioxidantes. Essa característica o torna bastante suscetível durante a sepse. $\mathrm{O}$ aumento de marcadores específicos de injúria cerebral está associado diretamente à mortalidade e é um preditor independente de sobrevida na terapia intensiva ${ }^{12}$.

$\mathrm{Na}$ avaliação neurológica do paciente em sepse grave, o enfermeiro deve atentar-se às disfunções para que seja realizada uma intervenção adequada, destacando-se a observação do nível de consciência, o qual pode apresentar-se como estado confusional, agitação psicomotora e delirium ${ }^{7}$. Os profissionais deste estudo demonstraram domínio nessa avaliação, uma vez que apontaram tais cuidados à avaliação do paciente séptico.

Grande parte dos pacientes sépticos apresenta edema intersticial, além da redução do surfactante, o que acarretará em desequilíbrio entre a ventilação e a perfusão pulmonar, resultando em hipoxemia, diminuição da complacência pulmonar e necessidade de ventilação mecânica para a adequada oxigenação tecidual ${ }^{13}$.

A competência e a habilidade para avaliação do padrão respiratório constituem-se essenciais aos enfermeiros que atuam em UTI, a fim de realizarem a monitorização respiratória eficazmente, contribuindo, assim, para a melhor evolução clínica do paciente em sepse grave.

Considerando toda a complexidade da repercussão respiratória desses pacientes, certas observações relevantes desse contexto parecem não ser analisadas por alguns profissionais deste estudo, como a importância da identificação precoce da relação $\mathrm{PaO}_{2} /$ $\mathrm{FiO}_{2}$ e a interpretação dos gases sanguíneos e das condições pulmonares à propedêutica clínica.

Todavia, a observação da frequência respiratória, da secreção traqueal, da saturação de $\mathrm{O}_{2}$ e do leucograma foi considerada na associação às alterações do padrão respiratório do paciente séptico.

A frequência de pacientes com lesão renal aguda em UTIs é comum, devido ao acometimento do sistema renal por tratamentos com o uso de drogas ou pela própria doença desenvolvida pelo indivíduo.

A oligúria apresenta-se como manifestação primária do sofrimento renal, e é ainda mais evidente na sepse. É recomendada essa observação na assistência ao paciente séptico por meio de protocolos específicos, devendo ser realizada com frequência nos processos de enfermagem desenvolvidos ${ }^{6}$.

Neste estudo, observou-se que tal ação é garantida pela maioria dos enfermeiros. Entretanto, outras observações igualmente importantes para a avaliação renal foram menos evidenciadas, como a avaliação dos níveis de ureia e creatinina. Isso se torna relevante, uma vez que a confirmação da lesão renal na sepse se dá a partir dessas análises ${ }^{6}$.

Percebe-se, portanto, que os enfermeiros devem aperfeiçoar sua assistência ao paciente séptico por intermédio da promoção de processos sistemáticos de avaliação clínica, a fim de garantir a adequada vigilância da função renal por meio da monitorização do volume de diurese, bem como dos demais indicadores clínico-laboratoriais de falência renal.

A introdução da terapia nutricional precoce auxilia na recuperação, diminui o tempo de internação, reduz a incidência de complicações infecciosas e influi diretamente na mortalidade dos pacientes graves, pois favorece a cicatrização, a melhora da resposta imunológica e a diminuição do estresse fisiológico ${ }^{14}$.

A abordagem terapêutica do paciente com diagnóstico de sepse continua sendo um desafio. O tratamento tem por objetivos: a manutenção de um suporte metabólico e cardiorrespiratório, a erradicação do processo infeccioso, o controle glicêmico e nutricional e o emprego de corticosteroides e anticoagulantes, além de medidas terapêuticas adicionais ${ }^{15}$.

Entretanto, parece que os enfermeiros, mesmo os que atuam há mais tempo em UTI, pouco privilegiam a avaliação do suporte nutricional do paciente séptico, haja vista que este estudo apontou que poucos relacionam a observação de níveis de 
glicemia à condição nutricional e à necessidade de início precoce de suporte nutricional. A maioria dos profissionais parece não conhecer o aporte calórico aos pacientes sépticos, pois menos de 30\% demonstraram conhecer as necessidades calóricas nas diversas fases da sepse.

Dessa forma, instituir e avaliar a eficácia dessa terapêutica é fundamental. É necessário que os enfermeiros tenham conhecimento sobre alterações metabólicas em paciente com sepse grave para que possam atuar de forma qualificada, propiciando ações e meios para que os pacientes recebam o aporte calórico necessário.

Conclui-se, pelos resultados encontrados neste estudo, que os profissionais de enfermagem têm dificuldade na identificação precoce das alterações sistêmicas causadas pela sepse grave relacionada às alterações hemodinâmicas, neurológicas, respiratórias, renais e nutricionais dos pacientes internados em UTIs adulto, o que pode estar relacionado com a falta de treinamento e de protocolos estabelecidos pelas instituições.

Fica evidente a necessidade de implementar protocolos para otimizar o serviço, com o intuito de desenvolver de forma assertiva e individualizada ações de enfermagem no cuidado ao paciente com sepse grave, pois o enfermeiro é o elo central da equipe, visto que planeja e coordena as ações de enfermagem apoiado no conhecimento técnico-científico.

\section{REFERÊNCIAS}

1. Kauss IA, Grion CM, Cardoso LT, Anami EH, Nunes LB, Ferreira GL, et al. The epidemiology of sepsis in a Brazilian teaching hospital. Braz J Infect Dis. 2010;14(3):264-70. http://dx.doi.org/10.1590/S1413-86702010000300011

2. Dellinger RP, Levy MM, Rhodes A, Annane D, Gerlach H, Opal $\mathrm{SM}$, et al. Surviving sepsis campaign: international guidelines for management of severe sepsis and septic shock, 2012. Crit Care Med. 2013;41(2):580-637.

http://dx.doi.org/10.1097/CCM.0b013e31827e83af

3. Levy MM, Fink MP, Marshall JC, Abraham E, Angus D, Cook D, et al. 2001 SCCM/ ESICM/ACCP/ATS/SIS International Sepsis Definitions Conference. Crit Care Med. 2003;31(4):1250-6.

http://dx.doi.org/10.1097/01.CCM.0000050454.01978.3B

4. Machado FR, Carrara F, Biasi AC, Bozza F, Lubarino J, Ferreira EM, et al. Prevalência e mortalidade por sepse grave e choque séptico em UTIs brasileiras. In: Anais do XI Fórum Internacional de Sepse; 2014 set. 18 e 19; São Paulo, Brasil. Disponível em: http://forumsepse.com. br//2014/assets/t//TL80.pdf. Acesso em: 10 jan. 2015.

5. Okamoto TY, Dias JCY, Taguti P, Sacon MF, Kauss AM, Carrilho CMDM, et al. Acute renal injury in patients with severe sepsis: prognostic factors. Sci Med. 2012;22(3):138-41.

6. Pinto CF, Watanabe M, Fonseca CD, Ogata Cl, Vattimo MFF. The sepsis as cause of acute kidney injury: an experimental model. Rev Esc Enferm USP. 2012;46(spe):86-90. http://dx.doi.org/10.1590/S0080-62342012000700013

7. Silva PS, Cordeiro FM, Gonçalves JM. Nurse's attention in the intensive care to the patient with signs of severe sepsis. Rev Enf UFPE. 2012;6(2):324-31. http://dx.doi.org/10.5205/reuol.2052-14823-1-LE.0602201210

8. Thibault R, Pichard C. Nutrition and clinical outcome in intensive care patients. Curr Opin Clin Nutr Metab Care. 2010;13(2):177-83. http://dx.doi.org/10.1097/MCO.0b013e32833574b9
9. Singer P, Pichard C, Heidegger CP, Wernerman J. Considering energy deficit in the intensive care unit. Curr Opin Clin Nutr Metab Care. 2010;13(2):170-6. http://dx.doi.org/10.1097/MCO.0b013e3283357535

10. Westphal G, Silva E, Salomão R, Machado F, Bernardo WM Sepse: ressuscitação hemodinâmica. Rev Assoc Med Bras. 2010;56(5):493-516 http://dx.doi.org/10.1590/S0104-42302010000500003

11. Brasil. Ministério da Saúde. Sociedade Beneficente Israelita Brasileira Hospital Albert Einstein. Instituto Latino Americano de Sepse. Programa de apoio ao desenvolvimento institucional do Sistema Único de Saúde. Controlando a infecção, sobrevivendo a sepse. Brasília: MS; 2012.

12. Siqueira-Batista R, Gomes AP, Calixto-Lima L, Vitorino RR, Perez MC Mendonça EG, et al. Sepsis: an update. Rev Bras Ter Intensiva. 2011;23(2):207-16. http://dx.doi.org/10.1590/S0103-507X2011000200014

13. Franca SA, Toufen C Jr, Hovnanian AL, Albuquerque AL, Borges ER, Pizzo VR, et al. The epidemiology of acute respiratory failure in hospitalized patients: a Brazilian prospective cohort study. J Crit Care. 2011;26(3):330.e1-8. http://dx.doi.org/10.1016/j.jcrc.2010.10.010

14. Associação de Medicina Intensiva Brasileira, Sociedade Brasileira de Infectologia, Sociedade Brasileira de Nutrição Parenteral e Enteral. Instituto Latino-Americano de Sepse. Diretrizes Clínicas na Saúde Suplementar. Sepse: nutrição. São Paulo: AMIB; 2011. p. 1-16.

15. Miranda RCC. Encefalopatia associada à sepse: disfunção comportamental, metabólica e mitocondrial em modelo de sepse abdominal. Dissertação (Mestrado) - Instituto Fundação Oswaldo Cruz. Rio de Janeiro: 2010. 